\title{
Silencing of decoy receptor 3 (DcR3) expression by siRNA in pancreatic carcinoma cells induces Fas ligand-mediated apoptosis in vitro and in vivo
}

\author{
JIAN ZHOU ${ }^{1 *}$, SHIDUO SONG $^{1 *}$, SONGBIN HE $^{1}$, ZHENXIN WANG $^{2}$, \\ BING ZHANG ${ }^{3}, \mathrm{DECHUN} \mathrm{LI}^{1}$ and DONGMING ZHU ${ }^{1}$ \\ Departments of ${ }^{1}$ General Surgery, ${ }^{2}$ Oncology and ${ }^{3}$ Nuclear Medicine, \\ The First Affiliated Hospital of Soochow University, Suzhou, Jiangsu 215006, P.R. China
}

Received April 1, 2013; Accepted July 3, 2013

DOI: $10.3892 /$ ijmm.2013.1437

\begin{abstract}
Decoy receptor 3 (DcR3) is abundantly expressed in human tumors and protects cells from a wide range of apoptotic stimuli. In this study, we demonstrate that DcR3 is overexpressed in pancreatic carcinoma cells, and that the pancreatic carcinoma cell lines, Panc-1 and SW1990, are resistant to Fas ligand (FasL)-mediated apoptosis. To further define the function of DcR3 in cell growth and apoptosis, we used small interfering RNA (siRNA) to knockdown the expression of the DcR3 gene in Panc-1 and SW1990 cells. Our results revealed that the silencing of DcR3 expression enhanced the inhibitory effects of FasL and reduced the capabiltiy of the cells for proliferation and colony formation in vitro. In addition, the downregulation of DcR3 modulated the cell apoptotic regulators, Fas-associated death domain (FADD), caspase-3 and caspase-8, thus triggering cell apoptosis. Furthermore, the knockdown of DcR3 inhibited the growth of Panc-1 tumor xenografts. Taken together, our findings indicate that DcR3 is important in cancer progression and may be a used as a potential therapeutic target for the gene therapy of pancreatic carcinoma.
\end{abstract}

\section{Introduction}

Human pancreatic carcinoma, a highly malignant cancer with a poor prognosis, is the sixth leading cause of mortality due to malignant disease in China and the fourth leading cause of cancer-related mortality in the United States $(1,2)$.

Correspondence to: Dr Dongming Zhu, Department of General Surgery, The First Affiliated Hospital of Soochow University, 188 Shizi Street, Suzhou, Jiangsu 215006, P.R. China

E-mail: zhoujian0612@gmail.com

*Contributed equally

Key words: decoy receptor 3, apoptosis, gene silencing, pancreatic carcinoma, small interfering RNA
The current literature indicates that the 5-year survival rate of pancreatic carcinoma patients remains $<5 \%$ and has not increased significantly over the past 20 years, partly due to the fact that pancreatic carcinoma cells are relatively resistant to chemotherapy and radiotherapy $(3,4)$. It has been suggested that resistance to Fas-Fas ligand (FasL)-mediated apoptosis may play an important role in the pathogenesis of pancreatic carcinoma; although human pancreatic adenocarcinoma cells express Fas and FasL, they are still resistant to Fas-mediated apoptosis (5).

As previously reported, a soluble decoy receptor 3 (DcR3) binds to FasL and inhibits FasL-mediated apoptosis. DcR3, also known as Tr6 or M68, is a member of the tumor necrosis factor receptor (TNFR) superfamily and maps to chromosome position 20q13, which is associated with gene amplification in various types of cancer. It shares sequence homology with osteoprotegerin (31\%), TNFR2 (29\%) and has relatively less homology with Fas (17\%) (6). It has been reported that DcR3 has 3 ligands: FasL, TNF-like molecule 1A (TL1A) and homologous to lymphotoxins, exhibits inducible expression, competes with herpes simplex virus glycoprotein D for HVEM, expressed by T lymphocytes (LIGHT) (7-9). DcR3 contributes to tumor growth by blocking apoptosis, impeding the immune response and inducing angiogenesis $(10,11)$. There is strong evidence indicating that DcR3 is overexpressed in a variety of human tumors, including cancers of lungs (6), colon (12) and liver (13), as well as gastric carcinoma (14) and malignant gliomas (15).

In this study, we examined the expression of DcR3 in pancreatic carcinoma tissues, serum and cell lines. Moreover, using small interfering RNA (siRNA) to silence the expression of DcR3, we investigated the effects of FasL-mediated apoptosis. Our findings indicated that DcR3 may be a potential target for gene therapy of pancreatic carcinoma.

\section{Materials and methods}

Clinical samples. Tissue samples from 50 pancreatic carcinoma patients were collected during surgical resections performed at the First Affiliated Hospital of Soochow University, Suzhou, China, from January 2008 to June 2012. Tumor tissues and 
adjacent non-tumor tissues were frozen immediately after surgical removal in liquid nitrogen and stored at $-80^{\circ} \mathrm{C}$. Serum for ELISA was obtained from cancer patients prior to surgery and serum from healthy individuals was used as the control. The patients had not received any pre-operative chemotherapy, radiotherapy or immunotherapy. All samples were obtained with patient consent and local ethics committee approval.

Lentiviral vectors for DcR3 siRNA. Three different siRNAs targeting DcR3 were designed using the DcR3 gene sequence (GenBank, NM-003823) as a template. The sequence with the most effective silencing effect was selected for subsequent experiments (data not shown). The recombinant lentivirus was synthesized and purified by Genechem Co., Ltd. (Shanghai, China).

Cell culture and transfection. The human pancreatic carcinoma cells, Panc-1 and SW1990, from the Shanghai Institute of Cell Biology (Shanghai, China) were maintained in Dulbecco's modified Eagle's medium (DMEM) or RPMI1640 (Gibco, Carlsbad, CA, USA) respectively, supplemented with $10 \%$ fetal bovine serum (Gibco) and $100 \mu \mathrm{g} / \mathrm{ml}$ each of penicillin-streptomycin (Invitrogen, Carlsbad, CA, USA) in $5 \% \mathrm{CO}_{2}$ at $37^{\circ} \mathrm{C}$.

In 6 -well plates, $5 \times 10^{4}$ cells/well were cultured overnight, then transfected with $10 \mu 1$ recombinant lentivirus of DcR3 siRNA (LV-RNAi) or mock lentivirus (LV-NC) using $5 \mu \mathrm{g} / \mathrm{ml}$ polybrene. Non-transfected cells were used as the blank controls.

Quantitative reverse transcriptase PCR ( $q R T-P C R)$. Total RNA from the tissues and cells was extracted using TRIzol reagent (Invitrogen). Single-stranded cDNA for a PCR template was synthesized from $10 \mu \mathrm{g}$ of total RNA using random primers and M-MLV reverse transcriptase (Takara, Dalian, China). The relative levels of target gene mRNA transcripts to those of the control (actin) were detemined by qRT-PCR. The primers used for PCR were as follows (forward and reverse): 5'-CTCTTCCTCCCATGACAC-3' and 5'-CTGGAAAGCC ACAAAGTC-3' for DcR3 (112 bp); 5'-AAGGAGTACACAGA CAAAGCCC-3' and 5'-GGTGATATTTACTCAAGTG-3' for Fas (684 bp); 5'-GCATTGGGCCTGGGGATGTTTCA-3' and 5'-TTGTGGCTCAGGGGCAGGTTGTTG-3' for FasL (344 bp); 5'-AGAGGTGGAGAACTGGGATT-3' and 5'-CCAA GGAAATGGGACAAA-3' for Fas-associated death domain (FADD) (119 bp); 5'-TTGGAACAAATGGACCTG-3' and 5'-ACAAAGCGACTGGATGAA-3' for caspase-3 (278 bp); 5'-TGAACCCAAGAGGTCAAG-3' and 5'-AGAAGGCAT AAAGCAAGT-3' for caspase-8 (192 bp); 5'-AGCGAGC ATCCCCCAAAGTT-3' and 5'-GGGCACGAAGGCT CATCATT-3' for actin (256 bp). The RT-PCR conditions were as follows: $10 \mathrm{~min}$ at $95^{\circ} \mathrm{C}$ for pre-heating, 40 cycles of $95^{\circ} \mathrm{C}$ for $15 \mathrm{~min}, 60^{\circ} \mathrm{C}$ for $1 \mathrm{~min}, 72^{\circ} \mathrm{C}$ for $30 \mathrm{sec}$, followed by extension at $72^{\circ} \mathrm{C}$ for $10 \mathrm{~min}$. The amplified segments were verified by electrophoresis on $2 \%$ agarose gels with ethidium bromide. The relative levels of mRNA transcripts to the control, actin, were calculated using the $2^{\Delta \Delta \mathrm{Ct}}$ method.

Cell viability assay. The effect of soluble FasL (sFasL) on cancer cell viability was measured using the Cell Counting
Kit-8 (CCK-8). After $72 \mathrm{~h}$ of transfection, the cells were seeded in 96-well plates at $5 \times 10^{3}$ cells/well and treated with sFasL $(25 \mathrm{ng} / \mathrm{ml}$ ) for $24 \mathrm{~h}$. A total of $20 \mu \mathrm{l} \mathrm{CCK}-8$ (Dojindo Laboratories, Kumamoto, Japan) was added followed by incubation at $37^{\circ} \mathrm{C}$ for an additional $2 \mathrm{~h}$. An ultraviolet spectrophotometer was used to measure the absorbance of each well at $450 \mathrm{~nm}$, and the cell viability index was calculated as follows: (experimental OD value/control OD value) x100\%.

Colony formation assay. After $72 \mathrm{~h}$ of transfection, the cells were cultured at 500 cells/well in 6 -well plates at $37^{\circ} \mathrm{C}$ for 20 days. The cells were then treated with sFasL $(25 \mathrm{ng} / \mathrm{ml})$ for $24 \mathrm{~h}$ and fixed with methanol and stained with $1 \%$ crystal violet. The colonies containing $>50$ cells were counted using a microscope.

Cell proliferation assay. The effects of sFasL on cell proliferation were measured by EdU assay. After $72 \mathrm{~h}$ of transfection, the cells were seeded in 96-well plates at $5 \times 10^{3}$ cells/well and treated with sFasL $(25 \mathrm{ng} / \mathrm{ml})$ for $24 \mathrm{~h}$, then $50 \mu \mathrm{M}$ of EdU for additional $2 \mathrm{~h}$. The cells were fixed with $4 \%$ formaldehyde for $15 \mathrm{~min}$ and treated with $0.5 \%$ Triton X-100 for permeabilization. Subsequently, each well was stained with $100 \mu \mathrm{l}$ Apollo solution for $30 \mathrm{~min}$, then with $100 \mu \mathrm{l}$ Hoechst 33342 for $15 \mathrm{~min}$ and visualized under a fluorescence microscope. The cell proliferation rate was calculated as follows: $\left(\right.$ EdU $^{+}$cell number/Hoechst ${ }^{+}$cell number) $\mathrm{x} 100 \%$.

Cell cycle and apoptosis analysis. After $72 \mathrm{~h}$ of transfection, the cells were treated with sFasL $(25 \mathrm{ng} / \mathrm{ml})$ for $24 \mathrm{~h}$. For cell cycle analysis, the cells were collected and fixed in $70 \%$ ethanol at $4^{\circ} \mathrm{C}$ overnight, followed by staining with propidium iodide (PI) (BD Biosciences, Franklin Lakes, NJ, USA) and were kept in the dark at $4^{\circ} \mathrm{C}$ for $30 \mathrm{~min}$. The cell cycle was analyzed by FACSCalibur with CellQuest software. For apoptosis analysis, a volume of $100 \mu \mathrm{l}$ of cell suspension $\left(1 \times 10^{6}\right.$ cells $\left./ \mathrm{ml}\right)$ was labeled with $10 \mu \mathrm{l}$ of PI and $5 \mu \mathrm{l}$ of Annexin V/FITC (BD Biosciences). The cells were incubated in the dark for $15 \mathrm{~min}$ at room temperature and early apoptotic cells were assessed by FACSCalibur.

ELISA. Cell culture supernatants and serum from cancer patients were collected and DcR3 levels were measured using ELISA kits (R\&D Systems, Minneapolis, MN, USA) according to the manufacturer's instructions.

In vivo tumor model. Male BALB/C nude mice (4-6 weeks old and weighing 16-20 g) were purchased from the Shanghai Experimental Animal Center (Shanghai, China) and housed in a specific pathogen-free environment. Animal experiments were carried out in accordance with the Guide for the Care and Use of Laboratory Animals of Soochow University. To establish a tumor xenograft model, $2 \times 10^{6}$ Panc- 1 cells in $200 \mu \mathrm{l}$ saline were inoculated subcutaneously into the flanks of nude mice. When the tumors reached approximately 5-7 $\mathrm{mm}$ in diameter, the mice were randomly divided into groups and injected intratumorally with PBS, LV-NC or LV-RNAi/DcR3 $\left(1 \times 10^{7}\right.$ $\mathrm{pfu} / 50 \mu \mathrm{l})$ once per week for 4 weeks. The tumor size was measured every 5 days with calipers, and the tumor volumes were calculated according to the formula: $\mathrm{V}=\left(\mathrm{L} \times \mathrm{W}^{2}\right) \times 0.5$ 

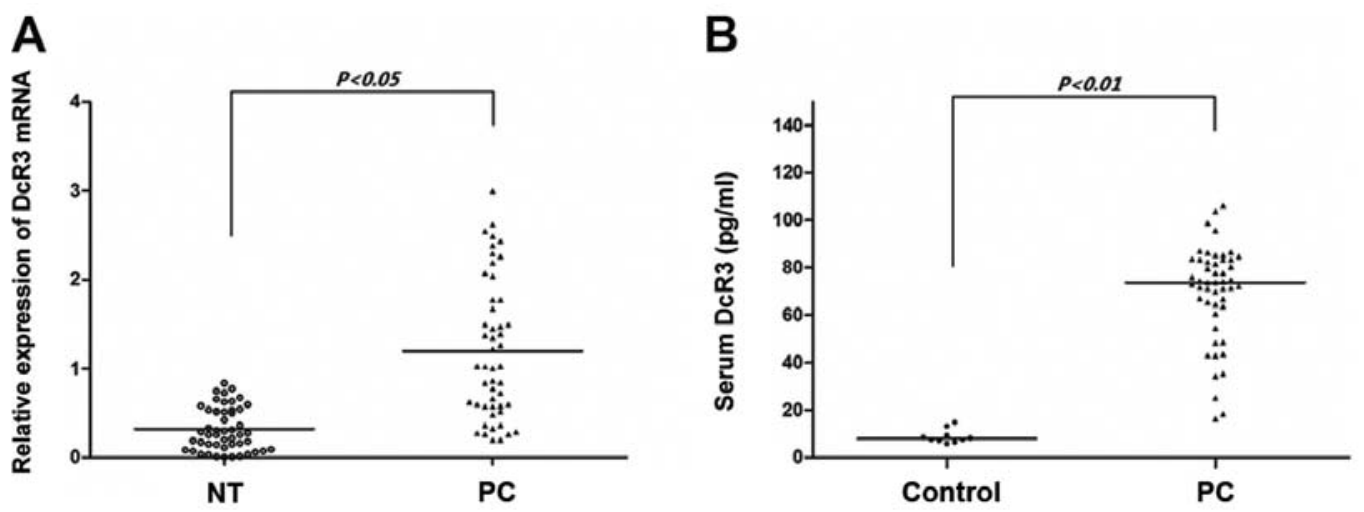

Figure 1. Expression levels of decoy receptor 3 (DcR3) in pancreatic carcinoma. (A) DcR3 mRNA expression in pancreatic carcinoma and non-tumor tissues (NT) by qRT-PCR. P $<0.05$ vs. NT. (B) The serum levels of DcR3 measured by ELISA. P $<0.01$ vs. control. Each dot represents a case. The data represent the means \pm SD of 3 experiments. PC, pancreatic carcinoma.
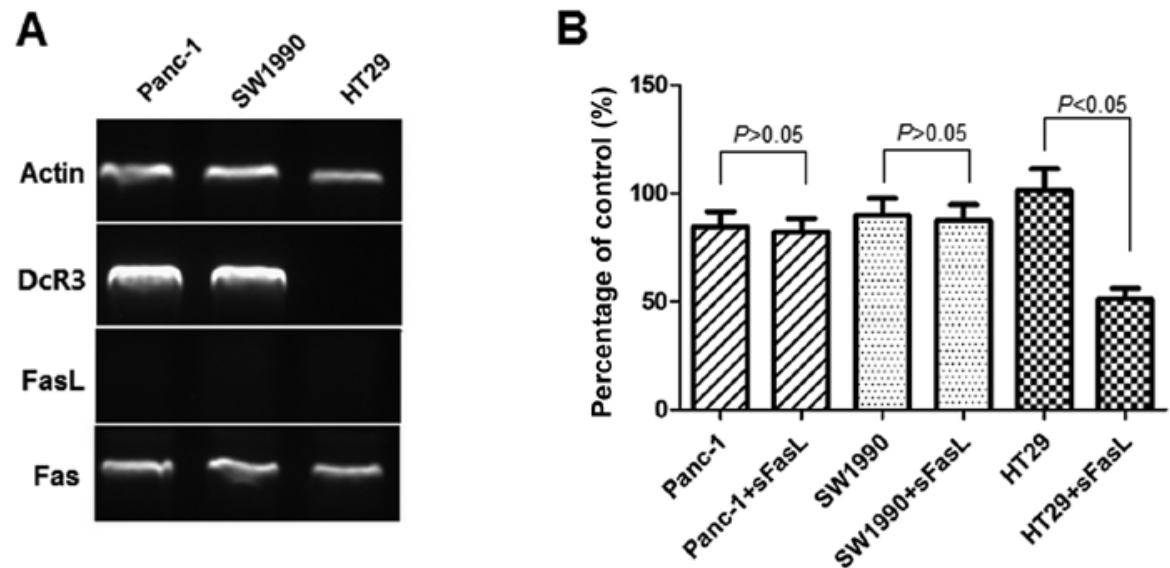

Figure 2. Expression of decoy receptor 3 (DcR3) and effect of soluble Fas ligand (sFasL) in Panc-1 and SW1990 cells. (A) DcR3, Fas, FasL mRNA expression was measured by RT-PCR analysis; actin was used as an internal reference and HT29 cells were used as negative controls for DcR3 expression. (B) The sensitivity of Panc-1 and SW1990 cells to sFasL was measured by CCK-8 assay. sFasL had no effect on Panc-1 and SW1990 cells, only on HT29 cells. "P<0.05 vs. HT29 cells not treated with sFasL. The data represent the means \pm SD of 5 experiments.

by measuring tumor length (L) and width (W). At the end of experiment, the tumors were dissected and weighed.

Statistical analysis. SPSS software version 16.0 was used for statistical analysis. Data are expressed as the means \pm SD. One-way analysis of variance (one-way ANOVA) or the t-test was performed for inter-group comparisons. A P-value $<0.05$ was considered to indicate a statistically significant difference.

\section{Results}

DcR3 is overexpressed in pancreatic carcinoma. In our cohort of 50 patients recently operated for pancreatic carcinoma, paired samples of tumor and non-tumor tissues were subjected to qRT-PCR (Fig. 1A). DcR3 mRNA was overpressed in the pancreatic carcinoma tissues compared with the non-tumor tissues $(\mathrm{P}<0.05)$. As DcR3 lacks a transmembrance sequence and is a soluble protein, we used ELISA assay to determine the serum levels of DcR3. As shown in Fig. 1B, the serum from the cancer patients had significantly higher levels of DcR3 than the serum from the controls $(\mathrm{P}<0.01)$. These results demonstrate that DcR3 is overexpressed in pancreatic carcinoma and that the protein expression of DcR3 is compatible with its mRNA expression.

Expression of DcR3 in pancreatic carcinoma cells and inhibitory effect of sFasL. The expression of FasL and that of its receptor, Fas, as well as that of DcR3, was assessed by RT-PCR (Fig. 2A). The Panc-1 cells and SW1990 cells expressed Fas and DcR3. The mRNA ratio of DcR3 to actin was $2.48 \pm 0.72$ for Panc-1 and $2.57 \pm 0.81$ for SW1990 cells. HT29 cells was used as the negative controls for DcR3 expression. We could not detect FasL expression by RT-PCR in the Panc-1 and SW1990 cells. To investigate whether DcR3 plays a role in the sensitivity to apoptosis mediated by FasL, we examined the effects of $24 \mathrm{~h}$ of exposure to sFasL $(25 \mathrm{ng} / \mathrm{ml})$ by CCK-8 assay (Fig. 2B). The results indicated that sFasL did not inhibit the cell growth of Panc-1 and SW1990 cells, but only that of HT29 cells, which do not express DcR3.

Silencing DcR3 expression by lentivirus-mediated RNA interference (RNAi). To investigate the role of DcR3 in the cell apoptosis mediated by FasL, the Panc-1 and SW1990 cells were transfected with recombinant lentivirus-mediated 

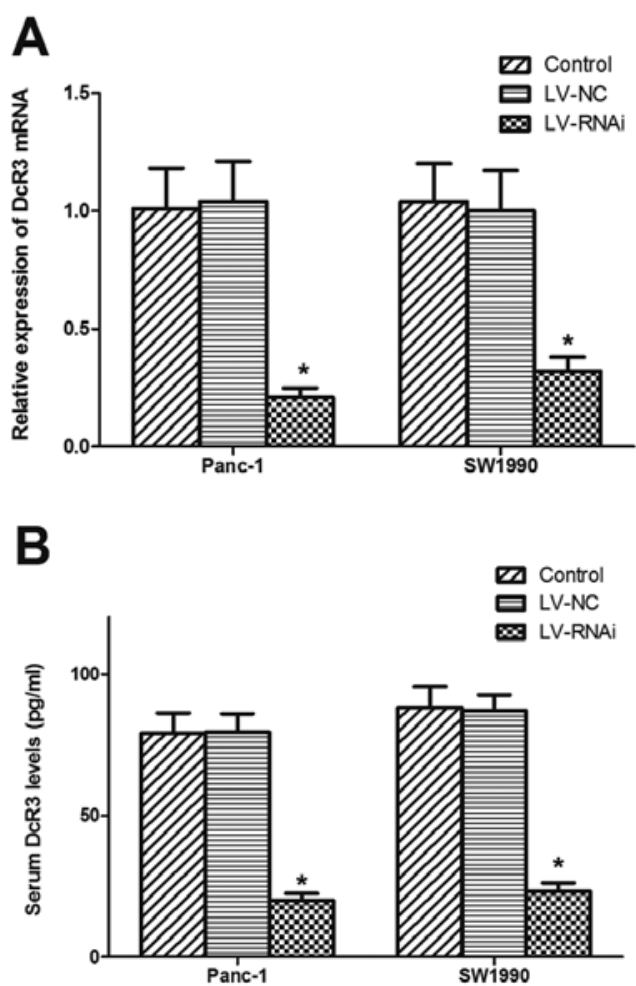

Figure 3. Silencing of decoy receptor 3 (DcR3) expression in Panc-1 and SW1990 cells transfected with LV-RNAi or LV-NC. (A) Relative expression of DcR3 mRNA measured by qRT-PCR. "P<0.05 vs. LV-NC-transfected cells. (B) Expression of DcR3 in the cell supernatants measured by ELISA. ${ }^{*} \mathrm{P}<0.05$ vs. LV-NC-transfected cells. The data represent the means $\pm \mathrm{SD}$ of 3 experiments.

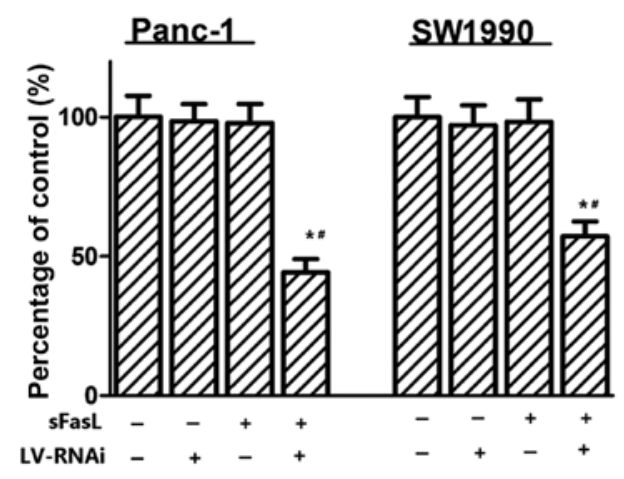

Figure 4. Effect of sFasL and/or decoy receptor 3 (DcR3) RNAi on cell viability by CCK- 8 assay. "P $<0.05$ vs. cells transfected with $L V-R N A i$ alone, ${ }^{\text {" }} \mathrm{P}<0.05$ vs. sFasL alone. The data represent the means $\pm \mathrm{SD}$ of 5 experiments.

RNAi targeting DcR3 and further used for functional analysis. As shown in Fig. 3A and B, the significant decrease in DcR3 expression was verified by RT-PCR and ELISA in the cells transfected with LV-RNAi.

Knockdown of DcR3 expression by RNAi enhances the effects of FasL. To explore the function of DcR3, cell proliferation was determined by CCK- 8 assays, colony formation and EdU assays. As shown in Fig. 4, there was no significant effect on cell viability without exposure to sFasL, whereas when the
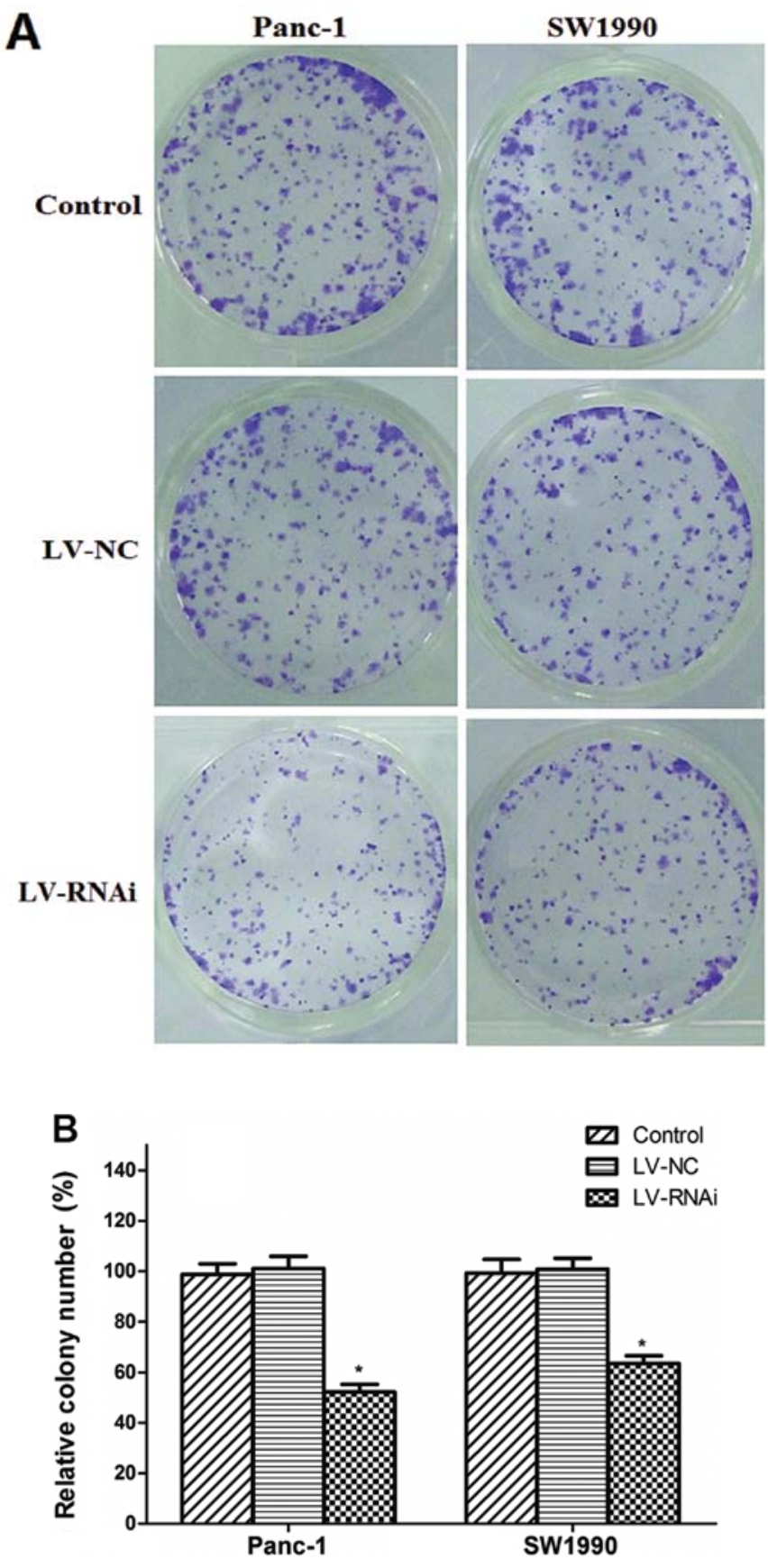

Figure 5. Effects of silencing decoy receptor 3 (DcR3) expression on colony formation. (A) Representative results of colony formation. (B) Histogram indicates that the relative colony number of the LV-RNAi-transfected cells was reduced. " $\mathrm{P}<0.05$ vs. LV-NC-transfected cells. The data represent the means \pm SD of 3 experiments.

cells were transfected with DcR3 siRNA and treated with sFasL for $24 \mathrm{~h}$, cell growth inhibition was observed. An analysis of clonogenicity indicated that the LV-RNAi-transfected cells displayed much fewer and smaller colonies than the LV-NC-transfected cells (Fig. 5A). The relative colony number of LV-RNAi-transfected cells was reduced by almost 50 and 40\% in the Panc-1 and SW1990 cells, respectively (Fig. 5B). Similarly, we found that the number of EdU ${ }^{+}$LV-RNAitransfected cells was significantly reduced compared with the LV-NC-transfected cells $(\mathrm{P}<0.05)$. After silencing DcR3 

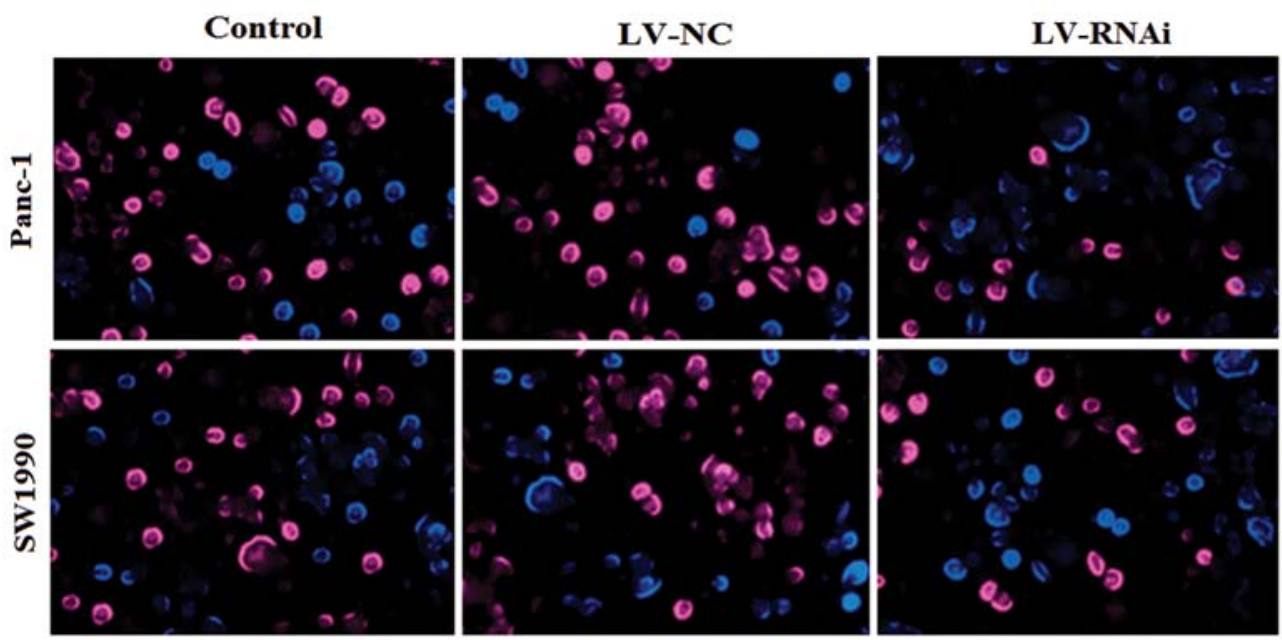

Figure 6. Effect of decoy receptor 3 (DcR3) RNAi on cell proliferation by EdU assay. The EdU-labeled cells (red fluorescence) and the Hoechst 33342-labeled cells (blue fluorescence) were examined under a fluorescence microscope (x200). After silencing DcR3 expression, the relative cell proliferation rate (LV-RNAi to LV-NC) was $41.46 \pm 4.25 \%$ for Panc-1 and $48.29 \pm 5.16 \%$ for SW1990 cells. A representative image (merged) is shown.
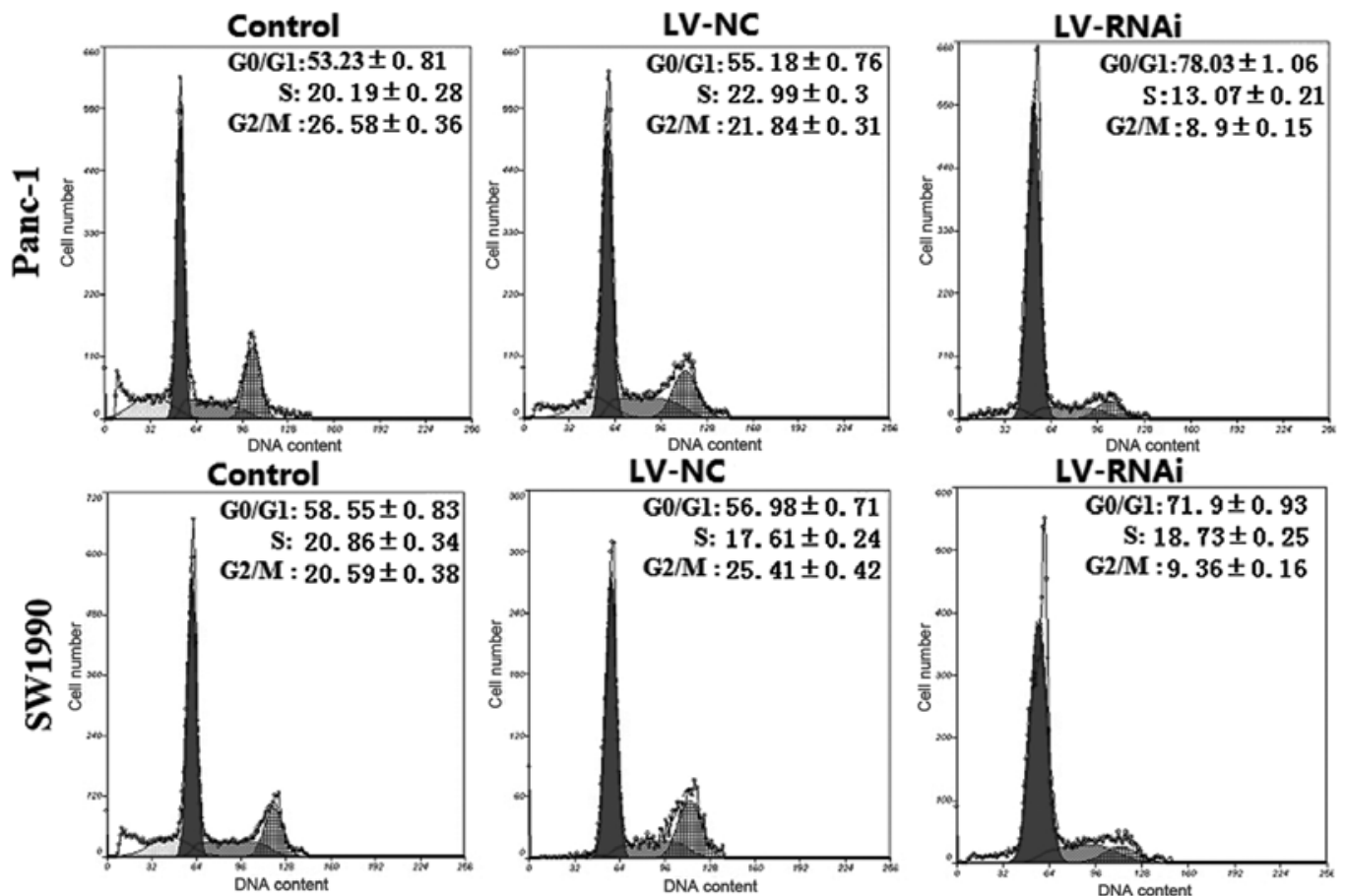

Figure 7. Cell cycle distribution was measured by flow cytometric analysis. A representative experiment of the 3 independent experiments is shown. The highest percentage of LV-RNAi-transfected cells was arrested at the G0/G1 phase. ${ }^{*} \mathrm{P}<0.05$ vs. LV-NC-transfected cells. The data represent the means \pm SD of three experiments.

expression, the relative cell proliferation rate (LV-RNAi- to LV-NC-transfected cells) was $41.46 \pm 4.25 \%$ for Panc-1 and 48.29 $\pm 5.16 \%$ for SW1990 cells (Fig. 6).

The cell cycle and apoptosis are associated with tumor cell growth and proliferation. Thus, we examined the effects of silencing DcR3 expression on the cell cycle and apoptosis by flow cytometry. As shown in Fig. 7, the percentage of G0/G1 phase LV-RNAi-transfected cells was $78.03 \pm 1.06 \%$ for Panc-1 and $71.9 \pm 0.93 \%$ for SW1990 cells, which was significantly higher than that of the LV-NC-transfected cells $(55.78 \pm 0.76 \%$ for Panc-1 and $56.98 \pm 0.71 \%$ for SW1990 cells;
$\mathrm{P}<0.05$, respectively). An analysis of apoptosis revealed that the number of apoptotic cells was significantly increased in the LV-RNAi-transfected cells compared with the LV-NCtransfected cells $(\mathrm{P}<0.05)$ (Fig. 8). These results suggest that the knockdown of DcR3 expression by RNAi enhances the apoptotic effects of FasL on pancreatic carcinoma cells.

Silencing DcR3 expression modulates the expression of cell apoptotic regulators. FADD, caspase- 3 and caspase- 8 are the regulators of the FasL-mediated apoptotic pathway. Following treatment with sFasL at $25 \mathrm{ng} / \mathrm{ml}$ for $24 \mathrm{~h}$, the relative mRNA 


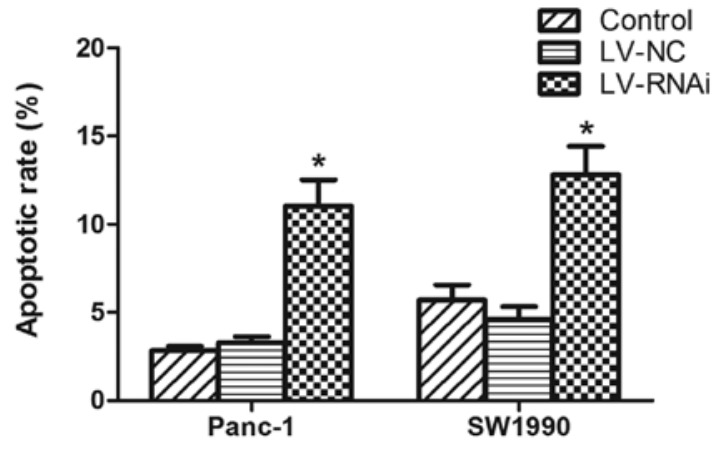

Figure 8 . Cell apoptosis was measured by flow cytometric analysis. ${ }^{*} \mathrm{P}<0.05$ vs LV-NC-transfected cells. The data represent the means \pm SD of 3 experiments.
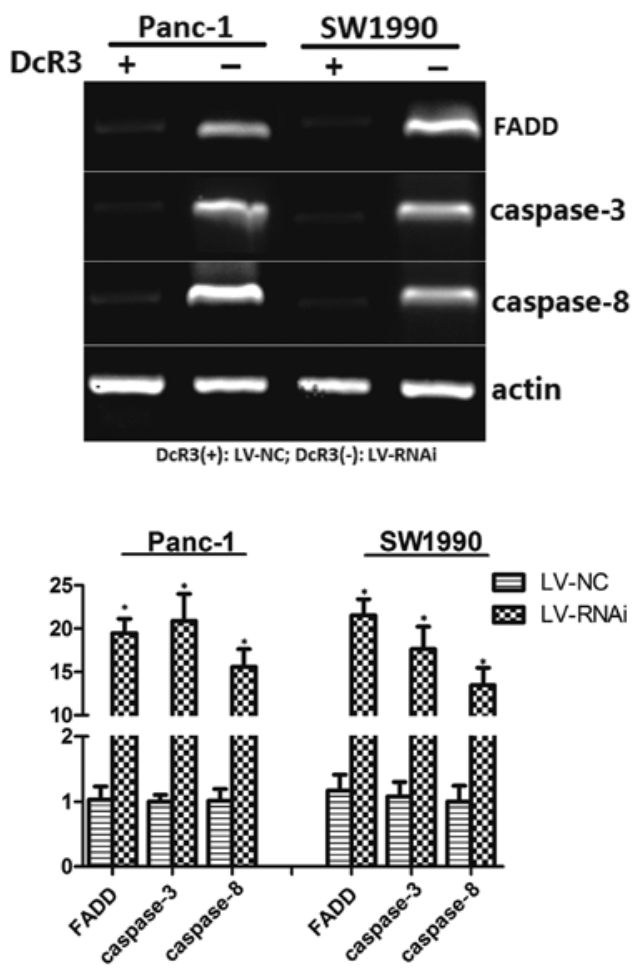

Figure 9. The expression of cell apoptotic regulators analyzed by RT-PCR Silencing decoy receptor 3 (DcR3) expression upregulated the expression of FADD, caspase- 3 and caspase- 8 . " $\mathrm{P}<0.05$ vs. LV-NC-transfected cells. The data represent the means \pm SD of 3 experiments.

levels of FADD, caspase- 3 and caspase- 8 to the control, actin, were determined by RT-PCR (Fig. 9). The results revealed that the cells transfected with LV-RNAi had an upregulated expression of FADD, caspase-3 and caspase-8. These results further support the hypothesis that silencing the expression of DcR3 modulates cell apoptotic regulators, thus triggering cell apoptosis.

Treatment with $L V-R N A i / D c R 3$ inhibits the growth of pancreatic carcinoma in vivo. As DcR3 plays a crucial role in pancreatic carcinoma, it may be used as a potential therapeutic target for the treatment of pancreatic carcinoma. BALB/C nude mice were inoculated with Panc-1 cells to establish subcutaneous tumor xenografts. The intratumoral injection of LV-RNAi/DcR3 significantly inhibited the growth of the
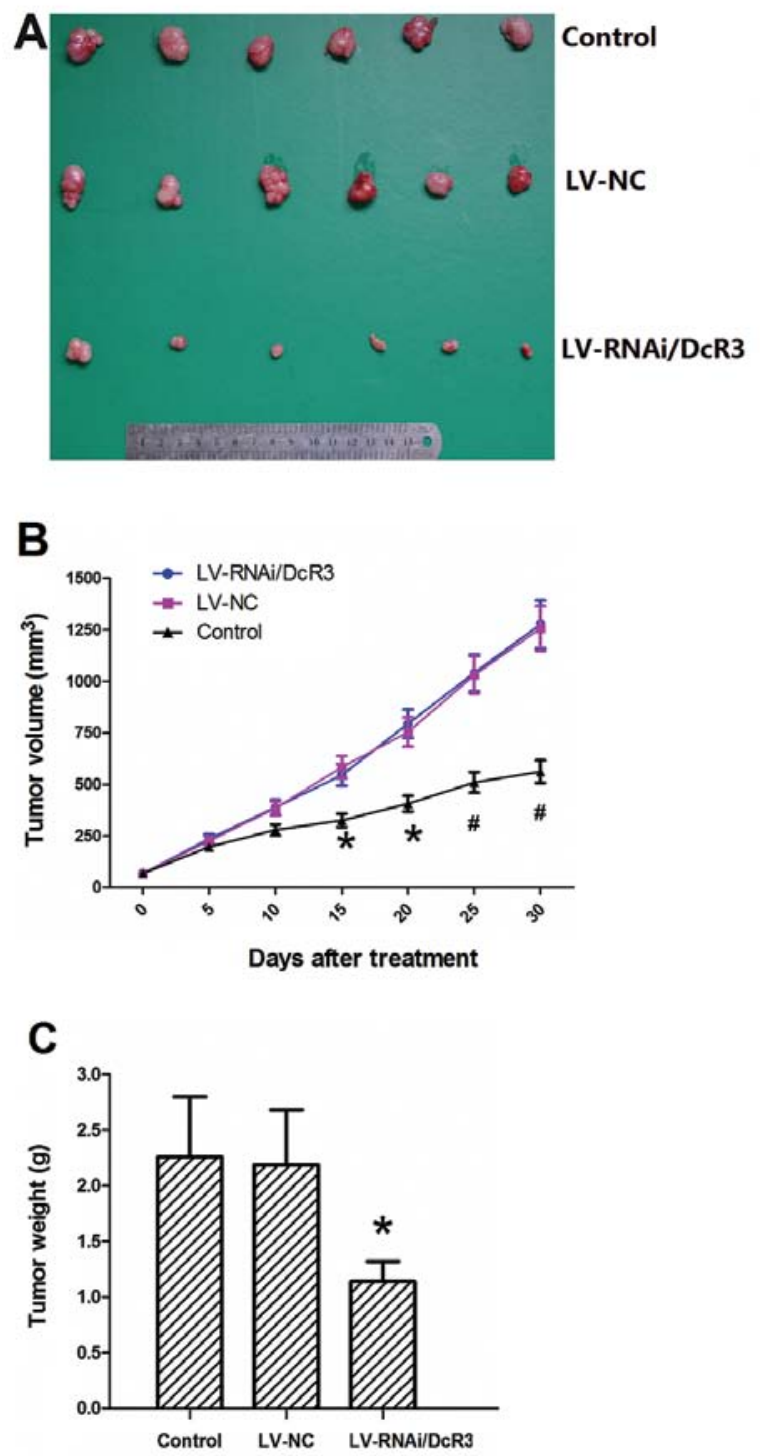

Figure 10. Effect of silencing decoy receptor 3 (DcR3) on the growth of pancreatic carcinoma cells in vivo. (A) Tumor morphology under different treatment conditions. (B) Tumor growth curve of different treatment groups. (C) Tumor weight of different treatment groups measured at sacrifice. Data are representative images or are expressed as the means $\pm \mathrm{SD}$ for tumor volumes and weight of each group ( $\mathrm{n}=6$ per group). ${ }^{*} \mathrm{P}<0.05$ vs. LV-NC-transfected cells, ${ }^{~} \mathrm{P}<0.01$ vs. $\mathrm{LV}-\mathrm{NC}$-transfected cells.

tumors (Fig. 10A). The growth curves of LV-RNAi/DcR3 and LV-NC became divergent after 15 days of treatment (Fig. 10B). The tumor weight at sacrifice in the LV-RNAi/DcR3 group was lower than that of the LV-NC group (Fig. 10C). These results indicated that LV-RNAi/DcR3 attenuated tumor growth and reduced tumor weight.

\section{Discussion}

In this study, we demonstrate that DcR3 is overexpressed in pancreatic carcinoma tissues, serum and cell lines. In our previous study, we demonstrated that the expression of DcR3 was associated with clinicopathological features, such as lymph node metastasis, tumor size and clinical stage (16). In this study, we also found that silencing DcR3 expression 
enhanced the apoptotic effects mediated by FasL in vitro and inhibited the tumor growth in vivo. DcR3 is a member of the TNFR superfamily and is regarded as a secreted molecule, as it lacks a transmenbrance sequence. Wu et al reported that $55 \%$ of patients with liver, gastric and colon carcinoma were serum DcR3-positive (17). The detection of DcR3 in serum offers an easy-to-access method for tumor diagnosis.

Apoptosis is a cell suicide mechanism which maintains a stable internal environment. The imbalance between cell proliferation and apoptosis plays an important role in the occurrence and progression of malignant tumors. FasL is mainly expressed in activated T cells and natural killer (NK) cells, and it induces apoptosis in target cells through the death receptor, Fas. The most common function of the FasL/Fas system is to mediate the killing of tumor cells by cytotoxic $\mathrm{T}$ cells However, studies have indicated that many tumors, including pancreatic carcinoma, are resistant to FasL/Fas-mediated growth inhibition signals, despite expressing Fas $(5,18)$. Similar results were obtained in this study using Panc-1 and SW1990 cells. Several mechanisms have been suggested to play a role in this phenomena, such as the downregulation of Fas (19), the upregulation of FasL (20) and the expression of soluble Fas (21). DcR3, which binds to FasL and inhibits FasLinduced apoptosis, may also play a role in the resistance to FasL/Fas-mediated growth inhibition signals.

DcR3 is a soluble decoy receptor belonging to the TNFR superfamily, which binds to FasL, LIGHT and TL1A. DcR3 can block the effects of FasL, TL1A and LIGHT by inhibiting the FasL-Fas, TL1A-death receptor 3 (DR3) and LIGHT-HVEM interaction (22-24). Evidence has shown that DcR3 not only protects tumor cells from apoptosis induced by FasL, LIGHT and TL1A, but also suppresses immune surveillance by blocking $\mathrm{T}$ cell costimulation mediated by TL1A and LIGHT $(25,26)$. Previous studies have demonstrated that DcR3 neutralizes the FasL-mediated apoptotic signal; therefore, we wished to determine whether the silencing of DcR3 expression enhances the apoptotic effects mediated by FasL.

In this study, we succeeded in silencing DcR3 using lentivirus-mediated RNAi targeting DcR3. We found that the knockdown of DcR3 expression in Panc-1 and SW1990 cells treated with sFasL inhibited cell viability, proliferation and clonogenicity. Flow cytometric analysis revealed that silencing DcR3 expression induced G0/G1 phase arrest in the cells transfected with LV-RNAi and induced apoptosis, compared with the cells transfected with LV-NC. These results suggest that the downregulation of DcR3 expression enhances the effects of FasL in Panc-1 and SW1990 cells. FADD, caspase-3 and caspase- 8 are the major regulators of the death receptor apoptotic pathway. FADD is a central adaptor molecule for Fas and forms a complex with Fas and pro-caspase-8 (27). Once caspase- 8 is released from the complex in an active form to transmit death signals to downstream caspase family members, such as caspase-3, apoptosis is induced. We found that the knockdown of DcR3 expression upregulated the expression of FADD, caspase-3 and caspase- 8 . These results further demonstrate that silencing DcR3 expression modulates cell apoptotic regulators, thus triggering cell apoptosis.

Pancreatic carcinoma is insensitive to radiotherapy and chemotherpy and anticancer therapy remains a major clinical challenge. Gene therapy has provided us with an alternative approach for the treatment of human cancer. The data presented in this study suggest the potential use of DcR3 as a therapeutic target. To the best of our knowledge, the anticancer effects of silencing DcR3 expression in vivo have not been reported to date. Our experiments on animals indicated that LV-RNAi/DcR3 suppressed the growth of pancreatic carcinoma xenografts in nude mice. The blocking of DcR3 may prove to be an effective therapeutic strategy, by supporting T lymphocytes and NK cells, which express FasL, to kill tumor cells.

In conclusion, this study demonstrates that DcR3 is overexpressed in pancreatic carcinoma tissues and serum. Silencing DcR3 by lentivirus-mediated DcR3 RNAi enhances the effects of FasL and inhibits tumor growth in vitro and in vivo. These findings indicate that DcR3 may be a potential therapeutic for the gene therapy of pancreatic carcinoma.

\section{Acknowledgements}

This study was supported by grants from the National Natural Science Foundation of China (no. 81201905), and the Post-Graduate Scientific Research Innovation Project of the Education Department of Jiangsu Province (no. CXLX11_0088), and Suzhou Science and Education Youth Health Foundation (no. 8WKQ0802), China.

\section{References}

1. Chen X, Ma S and Zhang Z: Analysis of clinical characteristics of pancreatic carcinoma in northern China. Pancreas 39: 1116-1118, 2010.

2. Auriemma WS, Berger AC, Bar-Ad V, et al: Locally advanced pancreatic cancer. Semin Oncol 39: e9-e22, 2012.

3. Vincent A, Herman J, Schulick R, Hruban RH and Goggins M: Pancreatic cancer. Lancet 378: 607-620, 2011.

4. Li D, Xie K, Wolff R and Abbruzzese JL: Pancreatic cancer. Lancet 363: 1049-1057, 2004.

5. Ungefroren H, Voss M, Jansen M, et al: Human pancreatic adenocarcinomas express Fas and Fas ligand yet are resistant to Fas-mediated apoptosis. Cancer Res 58: 1741-1749, 1998.

6. Pitti RM, Marsters SA, Lawrence DA, et al: Genomic amplification of a decoy receptor for Fas ligand in lung and colon cancer. Nature 396: 699-703, 1998.

7. Li W, Zhang C, Chen C and Zhuang G: Correlation between expression of DcR3 on tumor cells and sensitivity to FasL. Cell Mol Immunol 4: 455-460, 2007.

8. Bamias G, Siakavellas SI, Stamatelopoulos KS, Chryssochoou E, Papamichael C and Sfikakis PP: Circulating levels of TNF-like cytokine 1A (TL1A) and its decoy receptor 3 (DcR3) in rheumatoid arthritis. Clin Immunol 129: 249-255, 2008.

9. Gill RM and Hunt JS: Soluble receptor (DcR3) and cellular inhibitor of apoptosis-2 (cIAP-2) protect human cytotrophoblast cells against LIGHT-mediated apoptosis. Am J Pathol 165: 309-317, 2004.

10. You RI, Chang YC, Chen PM, et al: Apoptosis of dendritic cells induced by decoy receptor 3 (DcR3). Blood 111: 1480-1488, 2008.

11. Yang CR, Hsieh SL, Teng CM, Ho FM, Su WL and Lin WW: Soluble decoy receptor 3 induces angiogenesis by neutralization of TL1A, a cytokine belonging to tumor necrosis factor superfamily and exhibiting angiostatic action. Cancer Res 64: 1122-1129, 2004

12. Liang QL, Wang BR and Li GH: DcR3 and survivin are highly expressed in colorectal carcinoma and closely correlated to its clinicopathologic parameters. J Zhejiang Univ Sci B 10: 675-682, 2009.

13. Chen C, Zhang C, Zhuang G, et al: Decoy receptor 3 overexpression and immunologic tolerance in hepatocellular carcinoma (HCC) development. Cancer Invest 26: 965-974, 2008.

14. Wu Y, Guo E, Yu J and Xie Q: High DcR3 expression predicts stage pN2-3 in gastric cancer. Am J Clin Oncol 31: 79-83, 2008 . 
15. Arakawa Y, Tachibana O, Hasegawa M, Miyamori T, Yamashita J and Hayashi Y: Frequent gene amplification and overexpression of decoy receptor 3 in glioblastoma. Acta Neuropathol 109: 294-298, 2005

16. Zhou J, Song SD, Li DC, Zhu DM and Zheng SY: Clinical significance of expression and amplification of the DcR3 gene in pancreatic carcinomas. Asian Pac J Cancer Prev 13: 719-724, 2012

17. Wu Y, Han B, Sheng H, et al: Clinical significance of detecting elevated serum DcR3/TR6/M68 in malignant tumor patients. Int J Cancer 105: 724-732, 2003.

18. Song JH, Bellail A, Tse MC, Yong VW and Hao C: Human astrocytes are resistant to Fas ligand and tumor necrosis factor-related apoptosis-inducing ligand-induced apoptosis. J Neurosci 26 : 3299-3308, 2006.

19. Gratas $C$, Tohma $Y$, Barnas $C$, Taniere $P$, Hainaut $P$ and Ohgaki $H$ : Up-regulation of Fas (APO-1/CD95) ligand and down-regulation of Fas expression in human esophageal cancer. Cancer Res 58: 2057-2062, 1998.

20. Meng Y, Graves L, Do TV, So J and Fishman DA: Upregulation of FasL by LPA on ovarian cancer cell surface leads to apoptosis of activated lymphocytes. Gynecol Oncol 95: 488-495, 2004.
21. Abbasova SG, Vysotskii MM, Ovchinnikova LK, et al: Cancer and soluble FAS. Bull Exp Biol Med 148: 638-642, 2009.

22. Morishige T, Yoshioka Y, Inakura H, et al: Creation of a LIGHT mutant with the capacity to evade the decoy receptor for cancer therapy. Biomaterials 31: 3357-3363, 2010.

23. Takahashi M, Miura Y, Hayashi S, Tateishi K, Fukuda K and Kurosaka M: DcR3-TL1A signalling inhibits cytokine-induced proliferation of rheumatoid synovial fibroblasts. Int J Mol Med 28: 423-427, 2011.

24. Tsuji S, Hosotani R, Yonehara S, et al: Endogenous decoy receptor 3 blocks the growth inhibition signals mediated by Fas ligand in human pancreatic adenocarcinoma. Int J Cancer 106: $17-25,2003$

25. Migone TS, Zhang J, Luo X, et al: TL1A is a TNF-like ligand for DR3 and TR6/DcR3 and functions as a T cell costimulator. Immunity 16: 479-492, 2002.

26. Wan $X$, Zhang J, Luo $H$, et al: A TNF family member LIGHT transduces costimulatory signals into human T cells. J Immunol 169: 6813-6821, 2002.

27. Tourneur L, Buzyn A and Chiocchia G: FADD adaptor in cancer. Med Immunol 4: 1, 2005. 